\title{
The Effects of Paddy Soil Fungi Community Functional Diversity Under Organic Fertilizer Management Measures
}

\author{
Sun Dongye ${ }^{1, a}$, Liu Manqiang ${ }^{1, b}$, Hu Feng ${ }^{1, c}$ \\ ${ }^{1}$ College of Resources and Environmental Science, Nanjing Agricultural University, Nanjing 210095, \\ China. \\ asdybjb@163.com, bliumq@njau.edu.cn, ‘fenghu@njau.edu.cn
}

Key words: Organic fertilizer, Microflora, Functional diversity, BIOLOG.

\begin{abstract}
Paddy soil microbial community composition and functional diversity changes after a long-term fertilization were studied by BIOLOG. The results showed that the AWCD value eventually reached after a long-term fertilization was increased at some extent compared with the no fertilization. The diversity index results showed that the soil microbial community diversity was at a high level. The analysis for the utilization of carbon resources showed that communities were different on the utilization of carbon resource. Fungi and bacteria have obvious differences on the utilization of carbon resource and the activity ratio between fungi and bacteria was also different.
\end{abstract}

\section{Introduction}

The existing research evidences showed that fungi and bacteria's influence to the decomposition of organic matter and nutrient release was different greatly. However, they occupy different circulation way in the ecosystem, influenced by different factors[1,2]. The assimilation efficiency on the organic carbon of Bacteria and its carbon reserves were both lower than fungi,so the relative composition and functional changes of fungi and bacteria in the microbial community are associated with organic carbon reserves. However, there is few research about function of fungal diversity[3].

For the sustainable agriculture, the way of reasonable fertilizer management is the kernel[4]. The existing research have shown that the use of organic fertilizer had a significant effect on soil microbial community and composition [5]. Berthrong found that the diversity of bacteria and fungi community are increasing by using organic fertilizer for 20 years[6]. This research will use the Biolog to study the effect of different fertilization treatment on the functional diversity of fungal community.

\section{Materials and methods}

The sample for the research. The long-term trial was set in institute of red soil in JinXian county Jiangxi province,and materials were the quaternary red clay type with a breeding paddy soil in red soil development, whose profile pattern was A- P- W1- W2- G. The type of soil parent material was quaternary red clay, and the soil physical and chemical properties before the trial were shown in table 1. 
Table 1. the soil physical and chemical properties

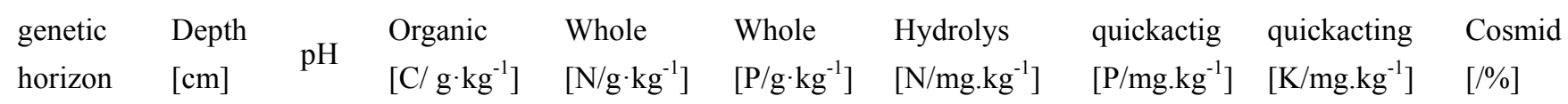

\begin{tabular}{llllllllll}
\hline A & $0 \sim 15$ & 6.9 & 16.22 & 0.952 & 1.021 & 143.7 & 10.3 & 125.1 & 55.8 \\
P & $15 \sim 24$ & 6.6 & 12.60 & 1.126 & 0.890 & 105.1 & 5.4 & 97.8 & 56.5 \\
W1 & $24 \sim 38$ & 6.2 & 3.751 & 0.692 & 0.618 & 37.0 & 1.6 & 54.0 & 58.4 \\
W2 & $38 \sim 90$ & 6.2 & 4.096 & 0.522 & 0.654 & 46.4 & 2.3 & 85.6 & 60.2 \\
\hline
\end{tabular}

Experiment design. A total of six treatment were established for the experiment and the design was shown in table 2 . The plot area was $60 \mathrm{~m}^{2}$ and arranged randomly with 3 repetitions.

Table2. The experimental design for organic fertilizer positioning in the red paddy soil

\begin{tabular}{crcc}
\hline Treatment & astragalus smicus & pig manure & straw \\
\hline MV & 22500 & & \\
VM & 22500 & 22500 & 4500 \\
VMS & 22500 & 22500 & 4500 \\
VS & 22500 & & \\
CF & & 160N, 16.4P, 100K & \\
CK & CK(no fertilizer) & \\
\hline
\end{tabular}

Note:Chemical fertilizer was applied to treatment $\mathrm{CF}$ with urea $\left(160 \mathrm{~kg} \mathrm{~N} \cdot \mathrm{hm}^{-2}\right)$,calcium-magnesium phosphate $\left(16.4 \mathrm{~kg} \mathrm{P} \cdot \mathrm{hm}^{-2}\right)$ and potassium chloride $\left(100 \mathrm{~kg} \mathrm{~K} \cdot \mathrm{hm}^{-2}\right)$ at early and late rice consistently during the experiment.

The collection and treatment of samples. The undisturbed soil samples of paddy soil arable layer were taken $(0-17 \mathrm{~cm})$ back to the laboratory and dried naturally in the middle of July, 2008. When the water content of soil reach to the plastic limit, you should cut gently large pieces of soil with your hand along the natural fractured surface,to exclude large plant residues and stones. And when it reaches $8 \mathrm{~mm}$, they should be sieved into the sealing bag after air dry constantly.

Assay method. $5 \mathrm{~g}$ soil sample (has been conversed into dry soil weight) was added to $45 \mathrm{~mL}$ $0.85 \%$ saline, and take them out after oscillation for $30 \mathrm{~min}$. And they were stood for $10 \mathrm{~min}$, then absorbed the soil solution, and after diluted 10 times, they were inoculated to the microwell plate, and the quantity of each hole is $125 \mu \mathrm{L}$. After inoculation for $1-7 \mathrm{~d}$, the OD value was measured under $590 \mathrm{~nm}$ wavelength. The tetrazolium blue indicator and carbon source should also be added into the microwell plate. In addition, the biological inhibitor streptomycin sulphate $(0.1 \mathrm{~g} / \mathrm{mL})$, chlortetracycline $(0.05 \mathrm{~g} / \mathrm{mL})$ were also needed to be added into it. The carbon sources included Dglucose, fructose,D- xylose, L- arabinose, cellobiose, cellulose, L- arginine, glycine, L- histidine,Lserine, L- $\alpha$-arginine, L- asp phthalein ammonia, L- sodium glutamate, sodium salicylate, gallic acid, tannic acid, vanillin, gelatin, gelatin, lignin and humic acid.

Data analysis. Microlog 4.2 software platform was used to transform the data into a Microsoft Excel format, and they were analyzed in the Excel and SPSS17.0 statistical softwares.

Results and analysis. There was significant difference for the utilization of carbon source under different fertilizer management. As can be seen from the figure 1, fungus's ability to use different 
carbon sources will become complicated when it exits different types of carbon source and culture time. The activity of VMS treatment (pig manure +astragalus smicus +straw) was the highest.
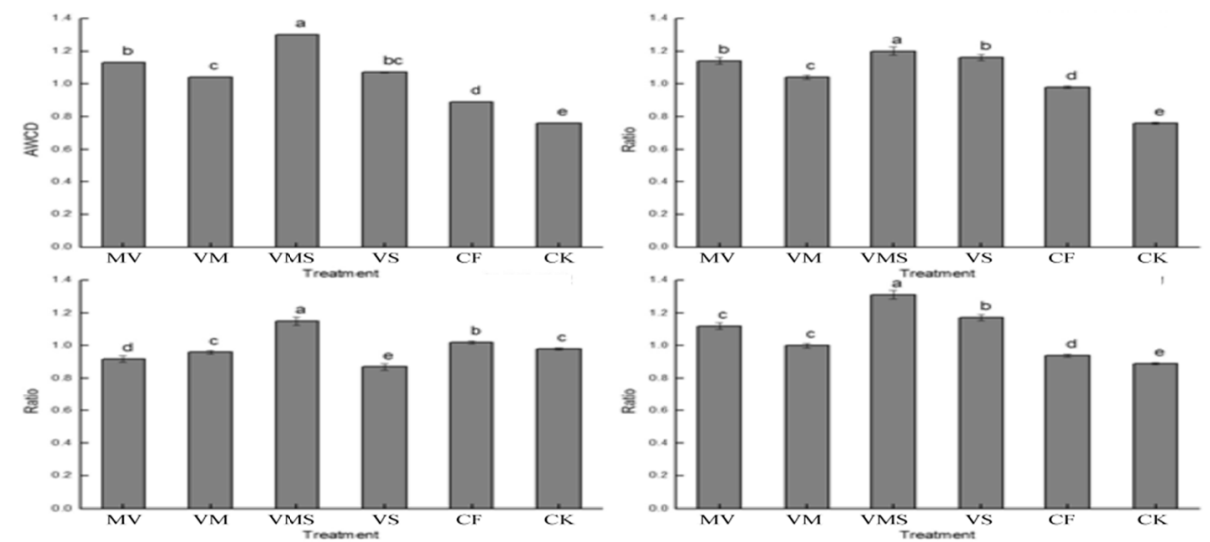

Fig. 1. Use of soil fungal communities under different organic management practices of various carbon sources

For fungi, the utilization ratio of amino acid and carbohydrate had significant difference among each treatment, and the VMS treatment was the most significant different and then was the MV treatment. The Fungi's utilization ratio of phenols and carbohydrate was similar to the utilization of amino acid and carbohydrate among different treatment. The Fungi's utilization of phenols and amino acids among different treatment was different markedly from the two utilization ratios above. The VMS treatment was the highest.

The fungal community composition expressed in AWCD standardized data was different from each other markedly (figure 2). The microbial community of MV and VM treatment had a strong ability to use lignin, glucose, xylose, arginine and gallic acid, however, the utilization ability to alanine and serine was weak. The microbial community of VMS and VS treatment had a strong ability to use tannic acid, sodium glutamate and arabinose, but the utilization ability to fructose and glycine was very weak. The microbial communities with $\mathrm{CK}$ treatment had a stronger utilization ability to fructose and cellobiose, but the utilization ability to sodium glutamate, arabinose, gallic acid, arginine, glucose and sodium salicylate was weak.

\section{Discussion and Conclusion}

Because of many problems with Biolog, Degens and Harris(1997) proposed a method to measure the microbial metabolism (breathing)by directly adding matrix to the soil[7]. First of all, 96 carbon sources in the Biolog existed a redundancy in a large extent[8].For the study which aimed to study the community function. So Degens and Harris(1997) extracted 36 kinds from the 83 kinds of carbon sources[7]. And it basically included the carbohydrates, amino acids and carboxylic acid, etc.

In addition, in recent the researchers began to pay close attention to fungal metabolic function, and the researches had shown that the carbon source utilization of fungal community was an important complement to bacteria community[8-9]. In conclusion, we adopted a compromised[10]method to determine the carbon source utilization of fungi, namely, we added low dilution degrees soil suspension to the micro tablet with 96 holes without any substrate and nutrient under the Biolog principle(on behalf of the original soil characteristics as much as possible, and 
forbid interfering the color of soil due to much soil particles), to measure the carbon source utilization of fungi.The experiment results showed that fungal community with different fertilizer treatments had different ability to use carbon source. The organic soil fungal community's ability to use carbon source was higher than others which applying chemical fertilizer or no fertilizer.

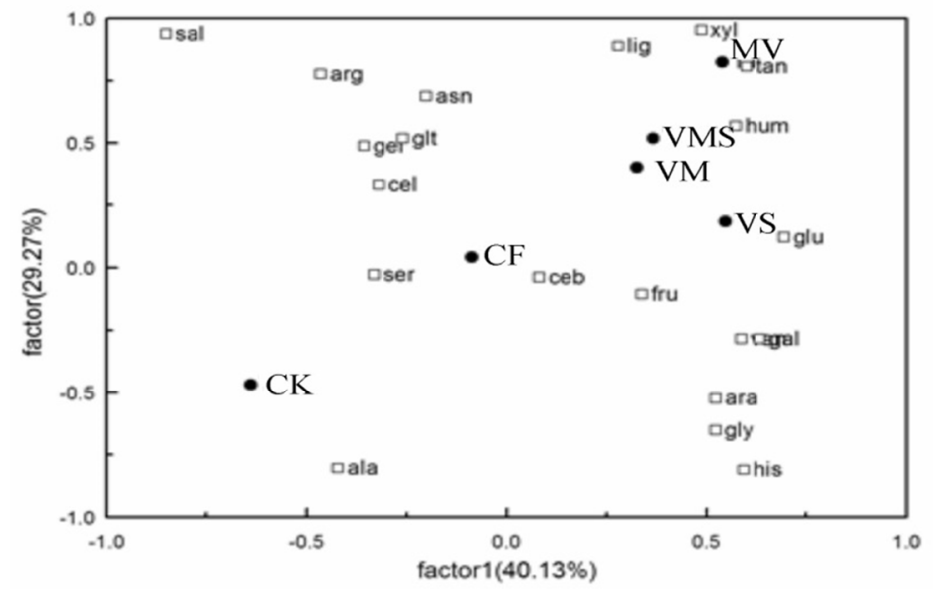

Fig.2. Using principal component analysis of 20 carbon source of the fungal community

\section{References}

[1] D.A. Wardle. Communities and ecosystems: linking the aboveground and belowground components, Princeton University Press, 2002.

[2] D.A. Wardle. G.W. Yeates. The dual importance of competition and predation as regulatory forces in terrestrial ecosystems: evidence from decomposer food-webs, Oecologia, 1993, 93(2): 303-306.

[3] V.L. Bailey., J.L. Smith and H.Bolton Jr., Fungal-to-bacterial ratios in soils investigated for enhanced C sequestration, Soil Biology and Biochemistry, 2002, 34(7): 997-1007.

[4] T.G. Ammari., R.Tahhan, S.Abubaker., et al., Soil salinity changes in the Jordan Valley potentially threaten sustainable irrigated agriculture, Pedosphere, 2013, 23(3): 376-384.

[5] P.Marschner., E.Kandeler., B.Marschner, Structure and function of the soil microbial community in a long-term fertilizer experiment, Soil Biology and Biochemistry, 2003, 35(3): 453-461.

[6] S. T.Berthrong., D.H.Buckley, L.E. Drink, water Agricultural Management and Labile Carbon Additions Affect Soil Microbial Community Structure and Interact with Carbon and Nitrogen Cycling,Microbial ecology, 2013: 1-13.

[7] B.P.Degens, J.A. Harris, Development of a physiological approach to measuring the catabolic diversity of soil microbial communities,Soil Biology and Biochemistry, 1997, 29(9): 1309-1320.

[9] A.T.Classen, S.I.Boyle, K.E.Haskins, et al., Community - level physiological profiles of bacteria and fungi: plate type and incubation temperature influences on contrasting soils,FEMS Microbiology Ecology, 2003, 44(3): 319-328.

[10] K.Ekschmitt, M.Liu, S.Vetter, et al., Strategies used by soil biota to overcome soil organic matter stability- Why is dead organic matter left over in the soil, Geoderma, 2005, 128: 167-176. 